\title{
TEVATRON ELECTRON LENS MAGNETIC SYSTEM
}

\author{
A.Ageev, I.Bogdanov, R.Hively ${ }^{1}$, S.Kozub, G.Kuznetsov ${ }^{3}$, E.Kashtanov, A.Martinez ${ }^{1}$, V.Pleskach, \\ H.Pfeffer ${ }^{1}$, A.Sery ${ }^{2}$, V. Shiltsev ${ }^{1}$, V.Sytnik, L.Tkachenko, D.Wolff ${ }^{1}$, S.Zintchenko, V.Zubko \\ Institute for High Energy Physics (IHEP), Protvino, Moscow region, Russia \\ ${ }^{1}$ Fermi National Accelerator Laboratory (Fermilab), Batavia, Illinois, USA \\ ${ }^{2}$ Stanford Linear Accelerator Complex (SLAC), Stanford, California, USA \\ ${ }^{3}$ Budker Institute of Nuclear Physics, Novosibirsk, Russia
}

\section{Abstract}

In the framework of collaboration between IHEP and FNAL, a magnetic system of the Tevatron Electron Lens (TEL) has been designed and built. The TEL is currently installed in the superconducting ring of the Tevatron proton-antiproton collider and used for experimental studies of beam-beam compensation [1].

\section{GENERAL DESCRIPTION OF MAGNETIC SYSTEM}

The longitudinal cross-section of the TEL magnetic system is shown in Fig. 1:

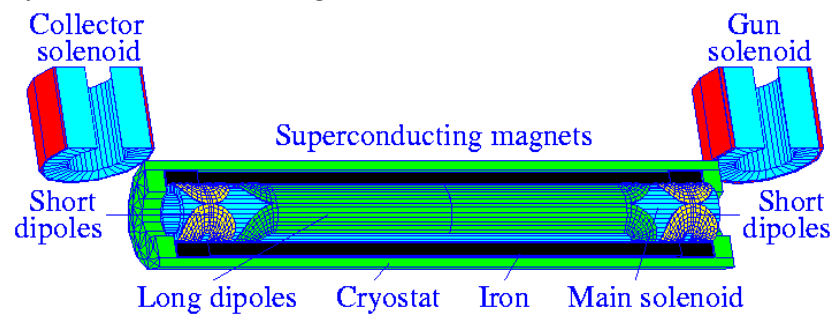

Fig. 1. Longitudinal cross-section of magnetic system.

The system consists of seven superconducting (SC) magnets (one large solenoid plus six steering dipoles) and two conventional solenoid magnets each equipped with corrector coils. An electron gun is placed in center of the first conventional solenoid and an electron beam collector in the second one. The electron beam is born on the electron gun cathode, transported through the interaction region in the strong solenoidal field of the SC solenoid and absorbed in the collector. The requirement of the field quality is that the magnetic field lines in the main SC solenoid are straight within $0.2 \mathrm{~mm}$ in both vertical and horizontal planes along 2-m length of the long dipoles.

\section{SUPERCONDUCTING MAGNETS}

The solenoid coil is constructed of a flat transposed cable consisting of $10 \mathrm{SC}$ wires (NbTi filaments in copper matrix) each $0.85-\mathrm{mm}$ diameter. The wire has $550 \mathrm{~A}$ critical current at $4.2 \mathrm{~K}$ and $5 \mathrm{~T}$ and $\mathrm{Cu} / \mathrm{SC}$ ratio of 1.38 . Dimensions of bare cable are $1.44 \times 4.64 \mathrm{~mm}^{2}$. The cable is wrapped by polyamide film of 0.03 -mm thickness with $1 / 3$ overlap. The main SC coil is wound on stainless steel tube of 151.4-mm diameter and 4-mm thickness. The frame insulation is three layers of polyamide film of 0.1mm thickness.

Six steering dipoles are placed on the outer surface of SC solenoid coil. Four pairs of 250-mm long coils form (short) lateral vertical and horizontal dipoles at each end of the solenoid. Two pairs of 2-meter long coils are placed in the central region of the SC solenoid. All these dipoles are for correcting the electron beam trajectory inside the magnetic system. The steering dipoles are wound of cable transposed from 8 wires of $0.3-\mathrm{mm}$ diameter. The wire has $50 \mathrm{~A}$ critical current at $4.2 \mathrm{~K}$ and $5 \mathrm{~T}$ and $\mathrm{Cu} / \mathrm{SC}$ ratio of 1.5. Dimensions of bare cable are $0.45 \times 1.48 \mathrm{~mm}^{2}$. The cable is wrapped by polyamide film of $0.03-\mathrm{mm}$ thickness with $1 / 3$ overlap. Lateral dipole cable is made of SC wires only. Current in central dipoles is small, and the cable has three SC wires and five $\mathrm{Cu}$ wires. The central dipoles have one layer; lateral dipoles consist of two layers and inter-layer spacer of 0.2-mm thickness.

Magnetic field calculations of the magnetic system were carried out using the MULTIC code [2]. SC solenoid coil together with steering dipoles is enclosed in a magnetic shield made of low-carbon steel. The shield is $48.5-\mathrm{mm}$ thick over the length of $270 \mathrm{~mm}$ and $38.5-\mathrm{mm}$ thick in the central part over 1.96-m length. The yoke reduces currents in steering coils, improves homogeneity of magnetic field inside solenoid aperture, compresses magnetic field lines at the ends of the coil block, and reduces stray fields. The outer surface of the iron has 16 grooves of $15-\mathrm{mm}$ width and $2-\mathrm{mm}$ depth. The grooves are need for passage of helium flow and they are also used for laying of cables for central and lateral dipoles. The yoke is produced of four nonlaminar equal-ring sector cylinders of $2.5-\mathrm{m}$ lengths. The yoke was tightened by two 5-mm thick stainless steel half-shells and then the shells were welded. The winding of solenoid with preliminary tension and compression of SC coil by bandage of the stainless steel half-shells allows one to reduce degradation and training of the SC coil. The main parameters of the TEL SC magnets are presented in Table. Computer calculations of the solenoid coil stress has been performed for all stages of winding and showed that cable tension during coil winding have to be $200 \mathrm{~N}$ and preload higher than $1 \mathrm{MPa}$ between coil and iron. Azimuth clearance of $0.5 \mathrm{~mm}$ between the ring sector parts of iron yoke during assembling is done. 
Table 1: Magnet Parameters

\begin{tabular}{lccccc}
\hline & Solenoid & Lateral & dipoles & Central & Dipoles \\
\hline Field direction & Longitudinal & Horizontal & Vertical & Horizontal & Vertical \\
Inner coil radius, mm & 76.00 & 100.0 & 103.7 & 100.0 & 103.7 \\
Outer coil radius, mm & 98.68 & 103.5 & 107.1 & 103.5 & 107.1 \\
Coil length, mm & 2500 & 270 & 270 & 1960 & 1960 \\
Number of layers & 14 & 2 & 2 & 1 & 1 \\
Total turn number & 7289 & 640 & 664 & 640 & 664 \\
Operating current, A & 1800 & 200 & 200 & 100 & 100 \\
Central field, T & 6.5 & 0.79 & 0.82 & 0.20 & 0.20 \\
Maximal field in coil, T & 6.5 & 2.2 & 2.2 & 0.5 & 0.5 \\
Stored energy, kJ & 950 & 1.2 & 1.3 & 0.9 & 1.1 \\
Inductance, H & 0.6 & 0.057 & 0.066 & 0.18 & 0.21 \\
Critical current $\left(\mathrm{B}_{\max }, 4.6 \mathrm{~K}\right), \mathrm{A}$ & 3000 & 640 & 640 & 540 & 540 \\
Critical temperature $\left(\mathrm{B}_{\max }, \mathrm{I}\right), \mathrm{K}$ & 5.3 & 7.1 & 7.1 & 8 & 8 \\
\hline
\end{tabular}

All SC coils and the magnetic shield are enclosed in a helium vessel. The vessel has 142.4-mm diameter and 4$\mathrm{mm}$ thick inner tube; the outer shell diameter is $324-\mathrm{mm}$ diameter and 5-mm thick and 2590-mm long. There is a box in the front of the helium vessel, which contains current leads, helium pipes and pipes going to a relief valve.

A thermal shield made of 2-mm thick copper sheet surrounds the helium vessel. The outer surface of the shield is covered with superinsulation and cooled by liquid nitrogen. Small radial gap between the inner tubes of the vacuum vessel and the nitrogen shield does not provide room for superinsulation and this part of the nitrogen shield is cooled by means of thermal conductivity.

The vacuum vessel has a 114-mm diameter and 2.1$\mathrm{mm}$ thick inner tube, a 480-mm diameter and 5-mm thick outer shell and is $2690-\mathrm{mm}$ long. Two boxes are attached in the head part of the vacuum vessel. A bigger box contains current leads, pipes for helium input-output and relief, connectors of potential wires. Smaller box has pipes for nitrogen input-output. The cold part of the magnetic system with mass of about $1350 \mathrm{~kg}$ is hung up to the vacuum vessel in two cross-sections with help of two vertical suspensions and two horizontal tension members in each of cross-sections. $10-\mathrm{mm}$ diameter tension members are made of titanium alloy. The cold mass is fixed axially by longitudinal titanium tension members and anchor fixed to the vacuum vessel.

The current leads of the main solenoid are designed for the maximum current of $1800 \mathrm{~A}$. Each current lead is a braid of 3500 copper wires of $0.13-\mathrm{mm}$ diameter placed in stainless steel tube of 14-mm inner diameter. The current leads are cooled by $0.125 \mathrm{~g} / \mathrm{s}$ of helium flow. The length of the cooled part is about $750 \mathrm{~mm}$. Heat leak through both $1800 \mathrm{~A}$ current leads to helium is equal to $4 \mathrm{~W}$. The current leads of the dipole magnets are designed for $200 \mathrm{~A}$ current. The current carrying element is $8-\mathrm{mm}$ diameter copper tube. Glass-cloth-base laminate washers connect six tubes in an assembly. The washers are pulled onto the tubes and are glued between them. The assembly is placed in 38-mm inner diameter stainless steel tube. The washers have grooves for $0.067 \mathrm{~g} / \mathrm{s}$ cooling helium gas flow. Heat leak to liquid helium through the current leads for all SC dipoles is $2 \times 1.4=2.8 \mathrm{~W}$.

Total static heat load onto the helium vessel is $12 \mathrm{~W}$ and $25 \mathrm{~W}$ onto the nitrogen thermal shield of the cryostat. The TEL cryostat is a part of the Tevatron magnet string cooling system. So, $24 \mathrm{~g} / \mathrm{s}$ of liquid helium pass through the channels in the yoke and ring gap between inner tubes of helium vessel and SC solenoid (20\% of the liquid helium flows through the ring gap).

During the change of current through the SC solenoid dynamic heat release occurs in the coil and other metal parts. Some heat is due to hysteresis in magnetization of the superconductor and the steel of the yoke. Heat is also provoked by eddy currents generated in inner stainless pipe, into the copper matrix of SC wires and in the yoke. To estimate the heat release, we used results of early measurements of properties of proper materials and the superconductor [3, 4]. Calculated power of the heat release in the main components of SC solenoid design is $1.5 \mathrm{~W}$ at the current ramp rate of $5 \mathrm{~A} / \mathrm{s}$ and $8.5 \mathrm{~W}$ at $18 \mathrm{~A} / \mathrm{s}$. Current ramp rate less than $10 \mathrm{~A} / \mathrm{s}$ is taken as a guideline value for the magnet excitation in order to limit the total heat load to liquid helium at $15 \mathrm{~W}$. In that case, the estimated heats of inner layer of the SC solenoid (at maximum magnetic field) and helium flow in the cryostat are 0.01 and $0.1 \mathrm{~K}$, respectively. Critical temperature of $\mathrm{SC}$ solenoid is $5.3 \mathrm{~K}$ at $1800 \mathrm{~A}$ and $6.5 \mathrm{~T}$ (see Table) and its temperature margin equals $0.6 \mathrm{~K}$ at helium temperature of $4.6 \mathrm{~K}$. For the dipole magnets critical temperature is $7.1 \mathrm{~K}$ and the temperature margin is bigger than in the SC solenoid.

\section{QUENCH PROTECTION}

The SC solenoid coil is not self-protected against resistive transition and fast quench detection and removal of stored energy to the external dump resistor must be taken. Simulation of quench spread through the coil was made for the case when quench was initiated at the end of the coil inner layer at the maximum current of $1800 \mathrm{~A}$, the quench detector threshold $1 \mathrm{~V}$, time delay $50 \mathrm{~ms}$, energy discharges on dump resistor $0.56 \mathrm{Ohm}$ and maximum voltage across the dump resistor less than $1000 \mathrm{~V}$. The quenching lasts about $2 \mathrm{~s}, 90 \%$ of stored energy (about 
$1 \mathrm{MJ}$ at $6.5 \mathrm{~T}$ ) dissipates in the dump resistor and $10 \%$ inside the cryostat, and the maximum temperature at the coil hot spot is about $270 \mathrm{~K}$.

The energy stored in SC dipoles is much smaller - about $1.3 \mathrm{~kJ}$ - and, in principle, one can allow all the energy to be dissipated in the coil if the quench is detected and the current interrupted. In that case hot spot temperature will not exceed $120 \mathrm{~K}$. But to lower risk of spreading the quench to the main solenoid, the scheme of quench protection with an external dump (as for the main solenoid) is used. Simulations of quench processes in lateral and central dipoles show that the maximum voltage across the dump resistor $(2 \mathrm{Ohm}$ for lateral and $8 \mathrm{Ohm}$ for central dipoles) is $400 \mathrm{~V}$; the maximum resistor's temperature rise is $80 \mathrm{~K}$. In this case the hot spot temperature does not exceed $43 \mathrm{~K}$ for lateral and $29 \mathrm{~K}$ for central dipoles. Quench protection circuits for each SC coil compare voltage across the coil with $L d I / d t$. If the difference exceeds $1 \mathrm{~V}$, a signal is sent to high current IGBT switches to disconnect the coil from power supply and allow to dump the coil current into the resistive load. Mechanical current breakers are installed in series with the solid state switches for redundancy.

\section{CONVENTIONAL MAGNETS}

The gun and collector solenoids have almost identical design. Each is wound of $8.25 \times 8.25 \mathrm{~mm}^{2} \mathrm{Cu}$ conductor with a $5.5-\mathrm{mm}$ diameter water hole. The solenoid has $0.4 \mathrm{~T}$ nominal magnetic field, $0.19-\mathrm{Ohm}$ electrical resistance and $18-\mathrm{mH}$ inductance. The coil has $250-\mathrm{mm}$ inner diameter, 474-mm outer diameter and 300-mm length. The solenoid coil consists of 17 pancakes (total number of turns 391), which are assembled on a common pipe of a 240-mm inner diameter. The pancakes are sorted in six sections, which have serial electrical current connection, while cooling water is supplied in parallel. Water temperature rise in the coil is $30^{\circ} \mathrm{C}$ at $0.7 \mathrm{MPa}$ pressure drop and nominal current of $340 \mathrm{~A}$.

The solenoids have a stainless steel flange at the end closer to the SC solenoid and an iron (low carbon steel) flange at the other end $(12 \mathrm{~mm}$ thick for the gun and 37 $\mathrm{mm}$ for the collector solenoid). To reduce stray fields, both solenoids have low carbon shields around the coil, which has average thickness of 12 and $20 \mathrm{~mm}$ for the gun and the corrector solenoids, respectively.

A tube of the magnetic field lines with initial radius of $5 \mathrm{~mm}$ and coming from the main SC solenoid to the collector solenoid is shown in Fig. 2. It needs about $100 \mathrm{~A}$ operating current in the short steering superconducting dipole in order for the electron beam to aim along the center of the warm solenoid.

Electron beam shape and position correctors are set inside each of the conventional solenoids. The corrector consists of four coils, which can be commutated either as a quadrupole or as two dipoles (vertical and horizontal). Each coil has layer shape geometry with $0.74^{\circ}$ inner and $40.04^{\circ}$ outer angles, $112.5-\mathrm{mm}$ inner radius and $8.6-\mathrm{mm}$ thickness. The length of coil is equal to $298 \mathrm{~mm}$. The coils were wound by $1-\mathrm{mm}$ diameter cooper wire and have 620 turns each. The dipole field is equal to $19 \mathrm{G} / \mathrm{A}$; the quadrupole field is equal to $6 \mathrm{G} / \mathrm{A} / \mathrm{cm}$.

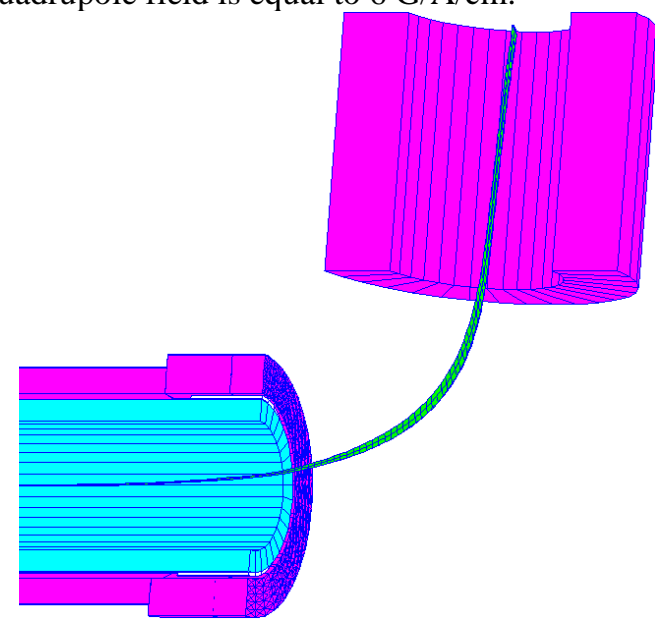

Fig. 2. Magnetic force tube from the SC solenoid to the collector solenoid in the case $\mathrm{B}_{\mathrm{SC}}=6.5 \mathrm{~T}$ and $\mathrm{B}_{\text {Coll }}=0.4 \mathrm{~T}$.

\section{OPERATIONAL EXPERIENCE}

In the first high-current test of the supercondicting solenoid, 6.6T was reached at the current ramp rate of $3 \mathrm{~A} / \mathrm{s}$ and after that the solenoid could not be quenched up to $6.7 \mathrm{~T}$ at 10,20 , and $30 \mathrm{~A} / \mathrm{s}$. The magnet quenches very quietly and does not consume much helium at the quench. We also found that inductance of the main solenoid varies from $0.56 \mathrm{H}$ at $0.5 \mathrm{~Hz}$ down to $0.19 \mathrm{H}$ at $200 \mathrm{~Hz}$. We think it is because of eddy currents in conductive pipes inside the coil, mostly in $2 \mathrm{~mm}$ copper nitrogen shield. Proper modifications introduced into the quench protection circuits led to more stable system operation. Magnetic field quality has been found within specifications [5]. The TEL has been installed in the Tevatron in February 2001, and been in operation since March and there were no quenches in the TEL at the typical operational field of $3.5 \mathrm{~T}$ in the main solenoid. It was found experimentally that electron beam can be steered to pass through the main solenoid if the gun solenoid field is in the range of $\mathrm{B}_{\mathrm{Gun}}=0.19-0.42 \mathrm{~T}$ for $\mathrm{B}_{\mathrm{SC}}=3.5 \mathrm{~T}$ (outside the range, the beam touches parts of the vacuum system, see [6]).

The magnetic system worked very reliably providing the control of the electron beam size and trajectory that allowed the first successful demonstration of the betatron tune shift of $980 \mathrm{GeV}$ protons in the Tevatron, see [1].

\section{REFERENCES}

[1] V.Shiltsev, et.al, this conference, see also Phys.Rev. ST-AB, v.2, 071001 (July 1999).

[2] L. Tkachenko, Preprint IHEP 98-28, Protvino (1998).

[3] A.Alexandrov, et al, IHEP Preprint 87-197 (1987).

[4] A.Baidakov, et.al, IHEP Preprint 92-77 (1992).

[5] K.Bishofberger, et.al, this conference.

[6] N.Solyak, this conference. 